\section{Desenvolvimento de forma farmacêutica líquida de uso oral, isenta de substâncias glicogênicas, com extrato fluido de Mikania glomerata Sprengel - Asteraceae (guaco)}

\author{
Lubi, N.C. ${ }^{1}$, Sato, M.E.O..$^{*}$, Gaensly, F. ${ }^{3}$ \\ ${ }^{1}$ Programa de Pós-graduação em Ciências Farmacêuticas \\ Universidade Federal do Paraná \\ ${ }^{2}$ Departamento de Farmácia, Setor de Ciências da Saúde \\ Universidade Federal do Paraná \\ ${ }^{3}$ Programa de Iniciação Científica, Universidade Federal do \\ Paraná
}

\section{Resumo}

Mikania glomerata Sprengel, Asteraceae (guaco) é tradicionalmente empregado como expectorante na forma de infusão, extrato fluido, tintura e xarope. Este trabalho teve como objetivo o desenvolvimento de uma forma farmacêutica líquida, isenta de substâncias glicogênicas, veiculando o extrato fluido desta, como substituto do xarope de guaco para pessoas, que por alguma restrição não possam ingerir sacarose ou açúcar invertido, componente dos xaropes em geral. Desenvolveu-se uma formulação líquida, definindo-se as concentrações de conservantes, agentes umectantes, edulcorantes e flavorizantes, testaram-se polímeros, espessantes usuais em processos tecnológicos, para reproduzir a viscosidade fornecida pelos xaropes. Analisou-se o comportamento reológico e uma avaliação preliminar das características físicas do produto. Dos polímeros testados, o alginato de sódio (1\%) foi o que apresentou melhores resultados frente aos parâmetros analisados, com comportamento reológico semelhante ao dos fluidos Newtonianos, e pH adequado ao uso oral, além da compatibilidade frente ao extrato fluido de guaco.

\begin{abstract}
Mikania glomerata Sprengel, Asteraceae (guaco), is traditionally used against cough as an infusion, a fluid-extract, a tincture or a syrup. The present investigation had as objective the development of a sugarless pharmaceutical liquid form to carry the guaco fluid extract, a substitute to the guaco syrup, it would specially help those who cannot ingest the glycogenic substances found in syrups. Liquid formulation was developed, in which the preservants concentration, the humectants agents, and sweeteners and flavors were defined, thickening polymers commonly used in technological processes in order to reduce the syrups viscosity were tested. The rheological behavior was analyzed and the preliminary evaluation of physical characteristic was done. Of all the tested polymers, the sodium alginate (1\%) showed itself the most stable, regarding the analyzed parameters. It had a rheological behavior similar to the Newtonian fluids and has adequate $\mathrm{pH}$ of oral use, as well as compatibility to the fluid guaco extract.
\end{abstract}

Mikania glomerata Sprengel, Asteraceae (guaco), vem de longa data sendo utilizada sob a forma de infusão, extrato fluido, tintura e xarope. Existem evidências científicas e populares, que o indicam como um fitoterápico útil para o combate de tosses de diversas origens ${ }^{1,2,3}$. Dependendo da natureza da droga, a administração via oral dos extratos fluidos são prejudicados pelo sabor e dosagem administrada, pois o uso excessivo pode conduzir à intoxicação. Desta forma, para facilitar a administração e dosagem convém incorporá-la a uma outra forma farmacêutica, sendo uma das mais empregadas o xarope, constituído por uma elevada concentração de sacarose ou açúcar invertido, o que proporciona à formulação viscosidade $\mathrm{e}$ dulçor. Esta forma farmacêutica é amplamente empregada em formulações expectorantes, entretanto, muitos pacientes que sofrem restrição quanto ao uso da sacarose, não podem utilizála. Desta forma, faz-se necessário desenvolver formulações isentas de açúcar, mas com propriedades edulcorantes e viscosidades semelhantes a esta.

No desenvolvimento de uma forma farmacêutica isenta de açúcar, pode-se substituir a sacarose por substâncias não glicogênicas doadoras de viscosidade ${ }^{4}$. Geralmente empregamse polímeros naturais (gomas, derivados celulósicos) ou sintéticos (derivados carboxivinílicos), que não sendo absorvíveis, constituem excelente veículo ${ }^{5}$. A viscosidade resultante com o uso destes polímeros, principalmente os derivados celulósicos assemelha-se à produzida pela sacarose e açúcar invertido ${ }^{6,7,8}$. Entretanto, estes polímeros não proporcionam às formulações o dulçor da sacarose e do açúcar invertido. Desta forma, para melhorar esta propriedade organoléptica, adicionam-se edulcorantes e agentes flavorizantes.

A viscosidade de uma solução serve para melhorar o paladar ou o escoamento, entretanto, soluções muito viscosas podem retardar a absorção de um fármaco, influenciando na velocidade de disponibilização do princípio ativo ${ }^{5,8,9}$. Líquidos que apresentam valores de viscosidade constante são fluidos newtonianos. Os fluidos não newtonianos mostram uma mudança na viscosidade com o incremento da tensão de cisalhamento. Serão chamados de plásticos se a viscosidade diminuir com o aumento da tensão de cisalhamento e se tornar constante num certo limiar, comportando-se como newtoniano; de pseudoplásticos se a viscosidade também diminuir, mas não obedecer a um limiar com o aumento da tensão de cisalhamento e dilatantes, quando o volume e a viscosidade aumentam com o incremento da tensão de cisalhamento ${ }^{9,10}$.

No desenvolvimento desta formulação, foram testados diversos polímeros doadores de viscosidade, edulcorantes e flavorizantes, analisando-se também, o comportamento reológico verificando a manutenção das características da formulação final, similares às dos xaropes e submetê-las a uma avaliação preliminar de estabilidade, analisando-se as características físicas. A tabela 1 e a figura 1 mostram o comportamento reológico e a figura 2 , a viscosidade da formulação com alginato de sódio $1 \%$ sem e com adição de extrato fluido 
Tabela 1. Comportamento reológico e viscosidade das formulações com alginato de sódio $1 \%$.

\begin{tabular}{ccccccc}
\hline $\begin{array}{c}\text { Velocidade } \\
\text { (r.p.m) }\end{array}$ & $\begin{array}{c}\text { Viscosidade } \\
(\mathrm{cP})\end{array}$ & $\begin{array}{c}\text { Viscosidade } \\
(\mathrm{cP})\end{array}$ & $\begin{array}{c}\text { Tensão de } \\
\text { cisalhamento } \\
\left(\mathrm{dyn} / \mathrm{cm}^{2}\right)\end{array}$ & $\begin{array}{c}\text { Gradiente de } \\
\text { cisalhamento } \\
(\mathrm{cm} / \mathrm{s})\end{array}$ & $\begin{array}{c}\text { Tensão de } \\
\text { cisalhamento } \\
\left(\mathrm{dyn} / \mathrm{cm}^{2}\right)\end{array}$ & $\begin{array}{c}\text { Gradiente de } \\
\text { cisalhamento } \\
(\mathrm{cm} / \mathrm{s})\end{array}$ \\
\hline & 1 & 2 & 3 & 4 & 5 & 6 \\
\hline 50 & 58,7 & 45,0 & 21,9 & 37,2 & 16,7 & 37,2 \\
60 & 56,0 & 43,0 & 26,0 & 46,5 & 20,0 & 46,5 \\
70 & 54,2 & 40,8 & 30,2 & 55,8 & 22,8 & 55,8 \\
80 & 53,6 & 39,4 & 34,9 & 65,1 & 26,0 & 65,1 \\
90 & 53,1 & 38,9 & 39,5 & 74,4 & 29,3 & 74,4 \\
\hline
\end{tabular}

Viscosidade (cP) da formulação com alginato de sódio (1) sem a adição de extrato fluido; (2) com a adição de extrato fluido, Tensão de cisalhamento $\left(\right.$ dyn $\left./ \mathrm{cm}^{2}\right)$ da formulação com alginato de sódio, (3) sem a adição de extrato fluido; (4) sem a adição de extrato fluido; (5) com a adição de extrato fluido, Gradiente de cisalhamento $(\mathrm{cm} / \mathrm{s})$ da formulação com alginato de sódio, (6) com a adição de extrato fluido.

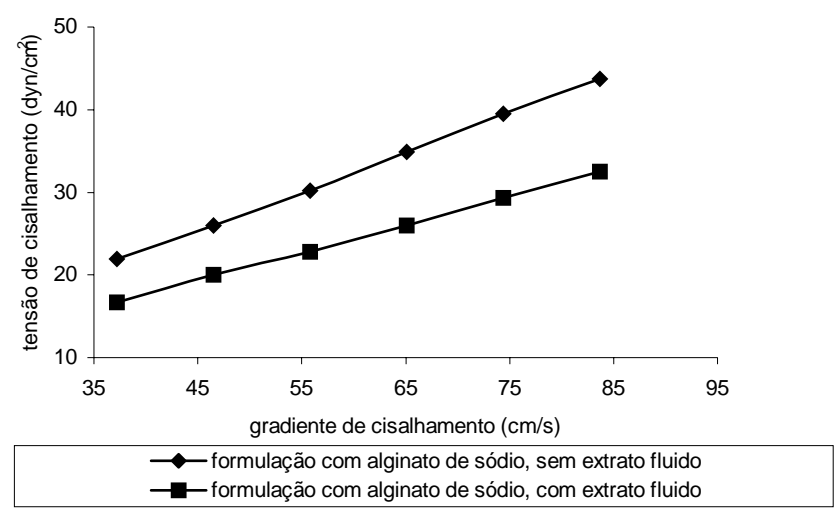

Figura 1. Comportamento reológico das formulações com $1 \%$ de alginato de sódio, sem e com extrato fluido de guaco.

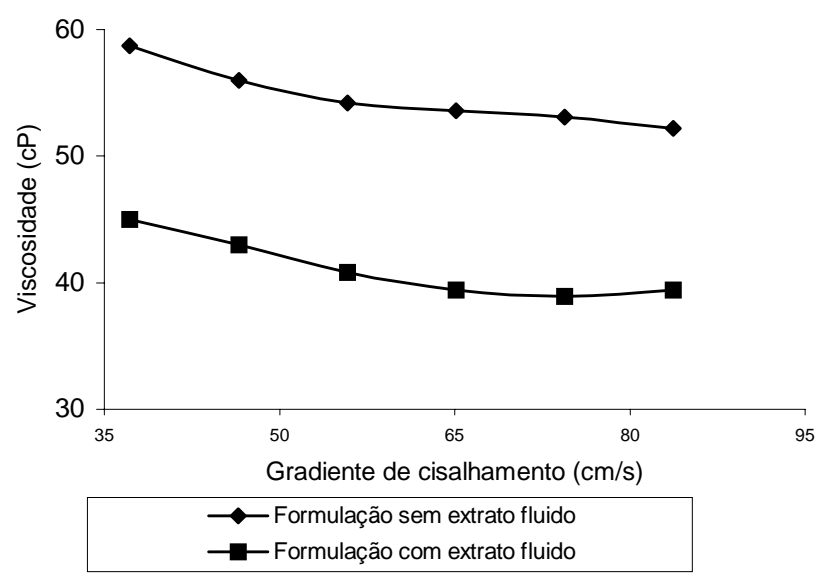

Figura 2 Viscosidade (cP) das formulações com $1 \%$ de alginato de sódio, sem e com extrato fluido de guaco

A tabela 2 refere-se às características organolépticas, aos valores de $\mathrm{pH}$ e viscosidade obtidos na avaliação preliminar das características físicas do produto, nas embalagens definitivas.
Tabela 2. Valores de pH e viscosidade na avaliação das características físicas do produto.

\begin{tabular}{|c|c|c|c|c|c|c|}
\hline \multirow{2}{*}{$\begin{array}{c}\text { Tempo } \\
\text { (dias) }\end{array}$} & \multirow{2}{*}{$\begin{array}{c}\text { Tempe- } \\
\text { ratura } \\
\text { de leitura } \\
\left({ }^{\circ} \mathrm{C}\right)\end{array}$} & \multirow{2}{*}{$\mathrm{pH}$} & \multirow{2}{*}{$\begin{array}{c}\text { Visco- } \\
\text { sidade } \\
(\mathrm{cP}), \\
60 \mathrm{rpm}\end{array}$} & \multicolumn{3}{|c|}{ Características organolépticas } \\
\hline & & & & cor & odor & sabor \\
\hline 0 & 25 & 5,46 & 40,0 & castanho & baunilha & adocicado \\
\hline 7 & 25 & 5,43 & 41,7 & - & - & - \\
\hline 14 & 25 & 5,41 & 40,8 & - & - & - \\
\hline 21 & 25 & 5,43 & 39,2 & - & - & - \\
\hline 60 & 25 & 5,40 & 40,0 & - & - & - \\
\hline 90 & 25 & 5,43 & 41,7 & castanho & baunilha & adocicado \\
\hline Ciclos & 25 & 5,46 & 120,0 & castanho & baunilha & adocicado \\
\hline
\end{tabular}

de temp.

No desenvolvimento da forma farmacêutica xarope, o principal doador de viscosidade com propriedades também edulcorantes, é a sacarose ${ }^{6,7,12}$ ou o açúcar invertido ${ }^{12}$ com comportamento reológico do tipo newtoniano. Entretanto, tanto a sacarose quanto o açúcar invertido são considerados substâncias glicogênicas ${ }^{5,7,12}$, pois a sacarose é absorvida e metabolizada em glicose, e o açúcar invertido, possui a glicose disponível ${ }^{13}$. Devido a isto, a recomendação para as pessoas que necessitem de uma dieta restrita em glicose é o uso de formulações com substâncias não glicogênicas ${ }^{5,6,7}$.

A viscosidade exerce um fator importante no desenvolvimento das formas farmacêuticas líquidas de uso oral, pois aumenta a estabilidade das formulações e facilita a administração ${ }^{4,7}$. O comportamento reológico para os líquidos ideais e xaropes são em geral do tipo newtoniano ${ }^{14}$, facilitando a administração e a posologia destes, os valores de viscosidade se mantém constantes independentes de alterações no sistema. Líquidos e xaropes que contém polímeros em sua composição podem apresentar comportamento reológico do tipo 
pseudoplástico, com diminuição da viscosidade pelo crescente cisalhamento, deste modo, para a administração e posologia devem ser agitados previamente para que se tornem mais fluidos ${ }^{8}$.

Os polímeros empregados, alginato de sódio, Carbopol 934 $\mathrm{P}^{\circledR}$, carboximetilcelulose sódica, goma xantana e Natrosol $250 \mathrm{HHR}^{\circledR}$, foram selecionados pelas suas propriedades de elevar a viscosidade, ampla faixa de $\mathrm{pH}$ de estabilidade das dispersões e a compatibilidade com a via de administração $0^{4,10,15}$. Foram avaliadas várias concentrações e utilizou-se a mínima para produzir um aumento de viscosidade e a manutenção destes valores na presença do extrato fluido de guaco.

Quanto à viscosidade da formulação sem o extrato fluido de guaco (tabela 1, figura 2), verificou-se que os valores diminuíram gradativamente, de 58,7 cP para 52,2 cP, conforme se aplicava um gradiente de cisalhamento, de comportamento pseudoplástica (tabela 1, figura 1$)^{10}$. Na formulação com extrato fluido de guaco observou-se que a viscosidade (figura 2), diminuiu de $45 \mathrm{cP}$ para $40 \mathrm{cP}$, com o aumento do gradiente de cisalhamento de $37,2 \mathrm{~cm} / \mathrm{s}$ para $55,8 \mathrm{~cm} / \mathrm{s}$, e manteve-se constante, na faixa de 39,4 cP, nos gradientes de cisalhamento maiores, $65,1 \mathrm{~cm} / \mathrm{s}$ e $83,7 \mathrm{~cm} / \mathrm{s}$. Este comportamento é característico dos plásticos de Bingham (tabela 1, figura 1) que, a partir do momento que se aplica uma alta força de cisalhamento, o material tende a fluir passando a ter um comportamento newtoniano, podendo ser explicado pelas interações moleculares e concentração do polímero na solução ${ }^{15}$. Observou-se também, que a adição de extrato fluido proporcionou a característica reológica própria dos xaropes e líquidos em geral.

$\mathrm{Na}$ avaliação preliminar das características físicas do produto, as formulações não apresentaram alterações quanto ao pH (tabela 2). Em relação à viscosidade (tabela 2), observouse que no tempo zero era de $40 \mathrm{cP}$ e após 90 dias de $41,7 \mathrm{cP}$ e quando submetidos aos ciclos de temperatura de $40 \mathrm{cP}$ para 120 cP. Balmaceda, cita que o alginato de sódio pode apresentar um comportamento dilatante com aumento de viscosidade, quando a formulação é submetida a condições extremas de temperatura, não sendo adequado para formulações líquidas, necessitando portanto, um controle de temperatura durante o processamento e estocagem deste produto.

Os edulcorantes, sacarina associada ao sorbitol, foram selecionados de acordo com as propriedades adoçantes e nas concentrações permitidas. A sacarina é $250-500$ vezes mais doce que a sacarose, mas deixa um resíduo amargo ${ }^{6,15}$, sendo que a associação com o sorbitol diminui este sensorial. Em relação ao uso do sorbitol, sabe-se que o mesmo é metabolizado em glicose e frutose. Entretanto, é considerado isento de risco, quando administrado em pequenas quantidades, devido à absorção lenta quando comparada à sacarose $\mathrm{e}^{15,16}$.

A essência de baunilha, agente flavorizante selecionado, intensificou o odor e sabor próprio do extrato, semelhante à baunilha ${ }^{1}$. Como agente dessensibilizante dos receptores do sabor amargo, próprio dos extratos, empregou-se a solução de mentol, que proporcionou um sabor agradável e refrescante à formulação. O sistema conservante foi selecionado, metilparabeno (Nipagin ${ }^{\circledR}$ ), considerando-se o $\mathrm{pH}$ de atividade e seu uso oral, o qual interferiu levemente nas características organolépticas quanto ao sabor, mas que foram mascarados pela solução de mentol.

As características organolépticas, cor, odor e sabor são relevantes para a adesão do paciente ao tratamento ${ }^{6,7}$. O reflexo nauseoso provocado por um gosto ou cheiro que provoque repugnância pode modificar a secreção dos sucos digestivos, dificultando a absorção do fármaco ${ }^{6}$. As formulações desenvolvidas apresentaram odor agradável característico do extrato (baunilha), sabor adocicado e cor castanha (tabela 2). A formulação desenvolvida com o alginato de sódio apresentou os melhores resultados quanto ao comportamento reológico, viscosidade, estabilidade, $\mathrm{pH}$ compatível com a via oral, compatibilidade com o extrato fluido, coadjuvantes farmacotécnicos, livre de precipitações e turvações..

Desenvolvimento de forma farmacêutica líquida oral, isenta de substâncias glicogênicas, presentes nos xaropes, mas com características organolépticas e parâmetros físicos similares a estes, torna-se possível com o uso adequado de polímeros e coadjuvantes farmacotécnicos, obtendo-se formulações estáveis, possibilitando que pessoas com restrições ao uso de componentes dos xaropes possam usufruir dos benefícios proporcionados, especificamente no caso deste estudo, das propriedades terapêuticas do guaco.

\section{Material e Métodos \\ Polímeros, coadjuvantes farmacotécnicos e fitoterápico}

Polímeros: alginato de sódio 1\%; carbômero (Carbopol $934 \mathrm{P}^{\circledR}$ ) 0,5\%; carboximetilcelulose sódica-média viscosidade (CMC) $1,5 \%$; goma xantana $1 \%$ e hidroxietilcelulose (Natrosol $250 \mathrm{HHR}^{\circledast}$ ) $0,5 \%$.

Coadjuvantes farmacotécnicos: essência de baunilha, glicerina, metilparabeno (Nipagin ${ }^{\circledR}$ ), propilenoglicol, sacarina sódica, solução alcoólica de mentol 10\%, sorbitol $70 \%$.

Fitoterápico: extrato fluido de guaco elaborado segundo procedimentos da Farm. Bras. ${ }^{17}$.

Formulações bases: Elaboradas de acordo com procedimentos farmacotécnicos e divididas em fases.

Fase A: polímero (de acordo com as especificações do item polímeros), água q.s.p. $100 \mathrm{ml}$.

Fase B: propilenoglicol 2\%, metilparabeno $0,2 \%$.

Fase C: glicerina 2,5\%, sorbitol (70\%) 5,0\%.

Fase D: sacarina sódica $0,1 \%$, essência de baunilha $0,1 \%$

Fase E:na formulação base(Fase $\mathrm{A}+\mathrm{B}+\mathrm{C}+\mathrm{D})$ adicionou-se $10 \%$ de extrato fluido de guaco

Fase F: neutralização com solução de hidróxido de sódio (50\%), para formulação com Carbopol $934 \mathrm{P}^{\circledR}$ para pH 5,5-6,0.

Avaliação do comportamento reológico: Formulações com os diversos polímeros na presença e na ausência do extrato fluido seguido ao processamento dos mesmos (tempo zero), realizado em viscosímetro rotacional (Brookfield DV II +, modelo RV, com redutor de amostra, spindle $21 \mathrm{e} 31$ ), temperatura de leitura, $25^{\circ} \mathrm{C}$. Condições para avaliação preliminar das características físicas do produto (embalagens definitivas): 
Ambientais (temperatura entre $25-30^{\circ} \mathrm{C}$, umidade relativa $60 \%$ ), período de 90 dias.

Ciclos de temperatura: 24 horas à temperatura de $40^{\circ} \mathrm{C}$, 24 horas em temperatura de (-) $5^{\circ} \mathrm{C}$ e 24 horas em condições ambientais (temperatura $25-30^{\circ} \mathrm{C}$, umidade relativa $60 \%$ ).

Avaliou-se as características organolépticas (cor,odor e sabor), viscosidade $\left(25^{\circ} \mathrm{C}\right)$ em viscosímetro rotacional (Brookfield DV II + , modelo RV, com redutor de amostra, spindle 21 e $\mathrm{pH}\left(25^{\circ} \mathrm{C}\right)^{14}$.

\section{Referências}

${ }^{1}$ Oliveira, F.; Oga, S.; Akisue, G.; Akisue, M.K. Parâmetros físicos e químicos e efeito antiedema dos extratos fluidos de guaco (Mikania glomerata Sprengel) e de guaco do mato (Mikania laevigata Schultz Bip .ex Baker). Rev. Farm. Bioquím. USP, v. 25, p. 50-54, 1985.

${ }^{2}$ Leite, R.M.G.; Leite, M.G.R.; de Souza, C.L.; da Silva, M.A.M.; Moreira, L.K.A.; Matos, F.J.A; Viana, G.S.B. Estudo farmacológico comparativo de Mikania glomerata Sprengel (guaco) e Justicia pectoralis Jacq. (anador) e Torresea cearensis Fr. (cumarú). Rev. Bras. Farm. v. 74, p. 12-15, 1993.

${ }^{3}$ Fierro, I.M.S.; Lopes, C.S.; Moura, R.S.; Fidalgo, C.B. Studies on the anti-allergic activity of Mikania glomerata. J.Ethnopharmacol., v. 66, p. 19-24, 1999.

4 Ansel, H.; Allen, J.R.; Popovich, N.G. Farmacotécnica-formas farmacêuticas e sistemas de liberação de fármacos. São Paulo: Premier, 6. ed., 2000.

${ }^{5}$ LeBlanc, P.P., et al. In:Buri, P. Disponibilização dos princípios activos no organismo, a partir das formas farmacêuticas destinadas à administração por via oral. Tratado de Biofarmácia e farmacocinética. Lisboa: Instituto Piaget, p. 61, 1999.

${ }^{6}$ Prista, L.; Alves, A.; Morgado, R. Técnica farmacêutica e farmácia galênica. Lisboa: Fundação Calouste Gulbekian, 3. ed., v. 12, 1990.

${ }^{7}$ Ansel, H.; Allen, J.R.; Popovich, N.G.; Pharmaceutical dosage forms and drug delivery systems. U.S.A.: Williams and Wilkins, 6. ed.,1995.

${ }^{8}$ Lachman, L.; Lieberman, J.L.; Kanig, J.L. Teoria e prática na indústria farmacêutica. Lisboa: Fundação Calouste Gullbekian, 2. ed., v. 2, 2001.

${ }^{9}$ Allen, J.R. Agentes de viscosidade para sistemas aquosos. International Journal Pharmaceutical Compounding, Brasileira,v. 6, 2000.

${ }^{10}$ Laba, D. Reological properties of cosmetics and toiletries. New York: Marcel Dekker, p. 9-31, 1993.

${ }^{11}$ Franchi, S.M. Contribuição ao estudo farmacognóstico da folha de Mikania glomerata Sprengel, Asteraceae. Dissertação Mestrado, Botânica, Setor de Ciências Biológicas, UFPR, Curitiba, 2000.

${ }^{12}$ Gennaro, A.R. Remington: The science and practice of pharmacy. $19^{\mathrm{a}}$ Ed. Pennsylvania: Mack Publishing Company, 19. ed., v. 2, p. 1447-995, 1995.

${ }^{13}$ Carstensen, J.T. Drug stability: principles and practices, New YorK: Marcel Dekker, 2. ed., v. 68, 1995.

${ }^{14}$ Jato, J.L.V. Tecnologia farmacêutica, formas farmacêuticas. Barcelona: Editorial Síntese, v. 2, 1997.
${ }^{15}$ Wade, A.; Weller, P. Handbook of pharmaceutical excipients. London: The Pharmaceutical Press, 2. ed. 1994.

${ }^{16}$ The United States Pharmacopea, XXIV, The National Formulary, Rockville, p.19, 1999.

${ }^{17}$ Silva, R.A.D. Pharmacopeia dos Estados Unidos do Brasil. São Paulo: Companhia Editorial Nacional, 1929.

\section{*Autor para correspondência:}

Profa. Dra. Mayumi Eliza Otsuka Sato

Laboratório de Tecnologia Farmacêutica

Departamento de Farmácia

Rua: Prefeito Lothário Meissner, 3400 - Jardim Botânico

CEP 80210-170 - Curitiba (PR)

E-mail:mayumi@ufpr.br 